\title{
Herbivore-Mediated Interaction Promotes the Maintenance of Trichome Dimorphism through Negative Frequency-Dependent Selection
}

\author{
$\operatorname{AUTHOR}(\mathrm{S}):$ \\ Sato, Yasuhiro; Kudoh, Hiroshi
}

\section{CITATION:}

Sato, Yasuhiro ... [et al]. Herbivore-Mediated Interaction Promotes the Maintenance of Trichome Dimorphism through Negative Frequency-Dependent Selection. The American Naturalist 2017, 190(3): E68-E77

\section{ISSUE DATE:}

2017-09

URL:

http://hdl.handle.net/2433/226924

\section{RIGHT:}

(c) 2017 by The University of Chicago.; The full-text file will be made open to the public on 01 September 2018 in accordance with publisher's 'Terms and Conditions for Self-Archiving'. 


\title{
Herbivore-Mediated Interaction Promotes the Maintenance of Trichome Dimorphism through Negative Frequency-Dependent Selection
}

\author{
Yasuhiro Sato* and Hiroshi Kudoh \\ Center for Ecological Research, Kyoto University, Hirano 2-509-3, Otsu, Shiga 520-2113, Japan \\ Submitted November 20, 2016; Accepted March 3, 2017; Electronically published June 7, 2017 \\ Online enhancements: appendix. Dryad data: http://dx.doi.org/10.5061/dryad.53k2d.
}

\begin{abstract}
A вSTRACт: Natural plant populations exhibit genetic variation in defense traits against herbivores. Despite a growing body of evidence for herbivore-mediated selection on plant defenses, we still know little about how genetic variation persists in antiherbivore defense traits. Here we present field and experimental evidence for herbivoremediated frequency-dependent selection that promotes the maintenance of trichome-producing (hairy) and trichomeless (glabrous) plants of Arabidopsis halleri subsp. gemmifera. First, in a natural population where the specialist leaf beetle Phaedon brassicae was prevalent, hairy plants were damaged less when the frequency of neighboring glabrous plants increased. Furthermore, temporal variation in the frequency of the two plant morphs showed that rarer morphs increased in frequency at the scale of 1-m-diameter patches between survey years. Using a mesocosm experiment, we demonstrated a rare-morph advantage for defense (leaf damage and herbivore abundance) and reproduction (flower and clone production) between hairy and glabrous plants in the presence of $P$. brassicae. However, this rare-morph advantage was not detected when beetles were absent, with glabrous plants having higher reproduction than hairy plants under these conditions regardless of frequency conditions. These findings highlight the overlooked but potentially critical role of herbivore-mediated apparent interaction in maintaining plant defense polymorphism.
\end{abstract}

Keywords: Arabidopsis halleri, apparent competition, frequency dependence, genetic variation, herbivory, plant defense.

\section{Introduction}

Herbivores remove substantial amounts of plant tissue in terrestrial ecosystems. Evidence is now accumulating that herbivores exert such strong selection pressure that the

\footnotetext{
* Corresponding author. Present address: Department of Plant Life Sciences, Faculty of Agriculture, Ryukoku University, Yokotani 1-5, Seta Oe-cho, Otsu, Shiga 520-2194, Japan; e-mail: sato.yasuhiro.36c@kyoto-u.jp.

ORCIDs: Sato, http://orcid.org/0000-0002-6466-723X.

Am. Nat. 2017. Vol. 190, pp. E67-E77. (C) 2017 by The University of Chicago. 0003-0147/2017/19003-57390\$15.00. All rights reserved.

DOI: $10.1086 / 692603$
}

evolution of plant defenses occurs on contemporary timescales (e.g., Zangerl et al. 2008; Agrawal et al. 2012; Züst et al. 2012; Sakata et al. 2014). If a trade-off exists between plant defense and plant growth, a single adaptive level of defense should be favored by a balance between the cost and the benefit of defense (Augner et al. 1991; Simms 1992; Tuomi et al. 1999). Thus, it will be difficult for natural selection to maintain genetic variation in defense levels unless it involves a stabilizing mechanism (Augner et al. 1991; Tuomi et al. 1999). However, natural plant populations indeed exhibit polymorphisms in defense traits against herbivores (e.g., Hughes 1991; Hare and Elle 2004; Kivimäki et al. 2007; Wise et al. 2009; Züst et al. 2012).

Negative frequency-dependent selection (NFDS) is, at least theoretically, a convincing mechanism for the maintenance of polymorphism, because it prevents rare morphs from becoming extinct (Ayala and Campbell 1974). NFDS on defense traits assumes that rare morphs are favored through learning behaviors (e.g., Greenwood 1984; Bond and Kamil 1998; Punzalan et al. 2005) or counteradaptation (e.g., Haldane 1949; Antonovics and Ellstrand 1984; Barrett 1988) by natural enemies. For example, in preypredator interactions, selective predation on abundant prey types mediates NFDS on cryptic coloration in a prey population (Bond and Kamil 1998; Punzalan et al. 2005). Several studies on host-parasite interaction provide suggestive evidence for frequency-dependent infection on multiple host genotypes (Antonovics and Ellstrand 1984; Dybdahl and Lively 1998; Brunet and Mundt 2000) and associated change of host genotype frequencies over time (Dybdahl and Lively 1998; Burdon and Thrall 1999; Siemens and Roy 2005). However, it has proven difficult to gather observational and experimental evidence for enemy-mediated NFDS in the same system (but see Dybdahl and Lively 1998).

In plant-herbivore interactions, studies on community ecology have shown that herbivory risk to an individual 
plant depends on not only its own traits but also the presence and frequency of other plant species or genotypes in the neighborhood (reviewed by Barbosa et al. 2009; Underwood et al. 2014). These associational effects phenomena have been recognized as a type of apparent competition between plant species (Barbosa et al. 2009). Specifically, recent studies hypothesized that associational effects influence the coexistence of multiple plant species or genotypes by mediating frequency-and/or density-dependent damage (Wise et al. 2009; Kim and Underwood 2015; Garrido et al. 2016; Sato and Kudoh 2016). For example, the tall goldenrod Solidago altissima receives reduced damage caused by a gallfly when candy cane morphs of this plant species are abundant in a neighborhood (Wise et al. 2009); however, whether this interaction leads to NFDS remains an open question.

Several species of the genus Arabidopsis exhibit genetic dimorphism of trichome-producing (hairy) and trichomeless (glabrous) plants (Hauser et al. 2001; Kivimäki et al. 2007; Kawagoe et al. 2011), and this dimorphism is governed by a few major genes (Grebe 2012). Trichomes of Arabidopsis have a major role in the physical resistance of plants to biotic and abiotic stresses (Handley et al. 2005; Kivimäki et al. 2007; Sletvold et al. 2010; Sletvold and Ågren 2012). On the other hand, trichomes impose a fitness cost in Arabidopsis (Mauricio 1998; Sletvold et al. 2010; Züst et al. 2011), raising a question about why the trichome dimorphism is maintained in nature. Likewise, Arabidopsis halleri (L.) O'Kane \& Al-Shehbaz subsp. gemmifera (Matsum.) O'Kane \& Al-Shehbaz (syn. Arabis gemmifera [Brassicaceae/Cruciferae]; abbreviated hereafter as $A$. halleri) also has genetic dimorphism of hairy and glabrous plants. Our previous research on A. halleri demonstrated that the specialist leaf beetle Phaedon brassicae Baly (Coleoptera: Chrysomelidae) causes frequency-dependent damage in a laboratory condition (Sato et al. 2014; Sato and Kudoh 2016), but herbivore-mediated NFDS remains unexplored in a natural population.

In this study, we tested the hypothesis that apparent interactions mediated by the leaf beetle $P$. brassicae promote the maintenance of hairy and glabrous $A$. halleri plants via NFDS. Three specific questions were addressed: (1) Do plant damage and fitness depend on the frequency of neighboring plant morphs? (2) Are the frequencies of rarer morphs restored to intermediate levels in the field over time? and (3) Could herbivores mediate a rare-morph fitness advantage for hairy and glabrous plants? To answer these questions, we first observed leaf damage, flower production, and temporal changes of the two morphs in a natural population where $P$. brassicae was a major insect herbivore on A. halleri. Then, in a common garden experiment, we manipulated both morph frequency and the presence or absence of herbivores to test whether the leaf beetle was an agent of NFDS between hairy and glabrous plants.

\author{
Material and Methods \\ Study Organisms
}

Arabidopsis halleri subsp. gemmifera is a self-incompatible perennial herb distributed across Japan and Far East Russia. Hairy morphs of this subspecies produce nonglandular trichomes on their leaf and stem surface, whereas glabrous morphs have no trichomes, except on their leaf margin (fig. $1 A, 1 B$ ). Hairy and glabrous phenotypes are associated with allelic variations in a trichome-related gene GLABROUS1 (GL1; Kawagoe et al. 2011). Plants usually start flowering from early April to May and are pollinated by small solitary bees and hoverflies. Flower production is a fine proxy of fruit production of $A$. halleri because fruit set is high $(>80 \%)$ in the field unless the flower is consumed by herbivores (Kawagoe and Kudoh 2010). After flowering, plants produce clonal rosettes on the main and lateral meristems (fig. $1 E$ ). Given this clonality, we designated individual plants as those with no vegetative connection to other plants in this study.

Phaedon brassicae is a crucifer-feeding pest that is distributed in Asia (Ôtake and Funaki 1958; Wang et al. 2007; fig. $1 C, 1 D)$. Adults are flightless and access host plants by walking (Ôtake and Funaki 1958; fig. 1D). Newly hatched larvae remain on the host plant on which the adults feed (Ôtake and Funaki 1958; fig. 1C); thus, differences in damage between individual plants are attributable to adult host choice. This species usually reaches the adult stage within 3 weeks of hatching, and adults survive for approximately 2 months (Wang et al. 2007). We used a laboratory-reared population that had been previously established and maintained on Chinese cabbage at $20^{\circ} \mathrm{C}$ and under $12 \mathrm{~L}: 12 \mathrm{D}$ conditions (Sato et al. 2014).

\section{Field Survey}

To investigate (1) whether plant damage and fitness depend on the frequency of neighboring plant morphs and (2) whether the frequencies of rarer morphs are restored to intermediate levels over time, we conducted a 4-year survey in a natural population of $A$. halleri located in Taka-cho, Hyogo Prefecture, Japan $\left(35^{\circ} 06^{\prime} \mathrm{N}, 134^{\circ} 56^{\prime} \mathrm{E}\right.$, altitude $\left.200 \mathrm{~m}\right)$. Field surveys were conducted for selected $A$. halleri patches along a creek (ca. $300 \mathrm{~m}$ ) that ran through the center of the study site. Vegetation is sparse along the creek, and no Brassicaceae plants other than $A$. halleri are present. No apparent microhabitat differentiation was observed between hairy and glabrous plants $(38 \mathrm{~cm}$ in median distance between the nearest two morphs; $<1.0 \%$ median difference in soil moisture nearby the two morphs, measured by the portable soil moisture meter DM-18 [Takemura Electric Works]). At this site, P. brassicae occurs mainly during the flowering season (April-May) and heavily infests A. halleri (Kawagoe and Kudoh 2010). 


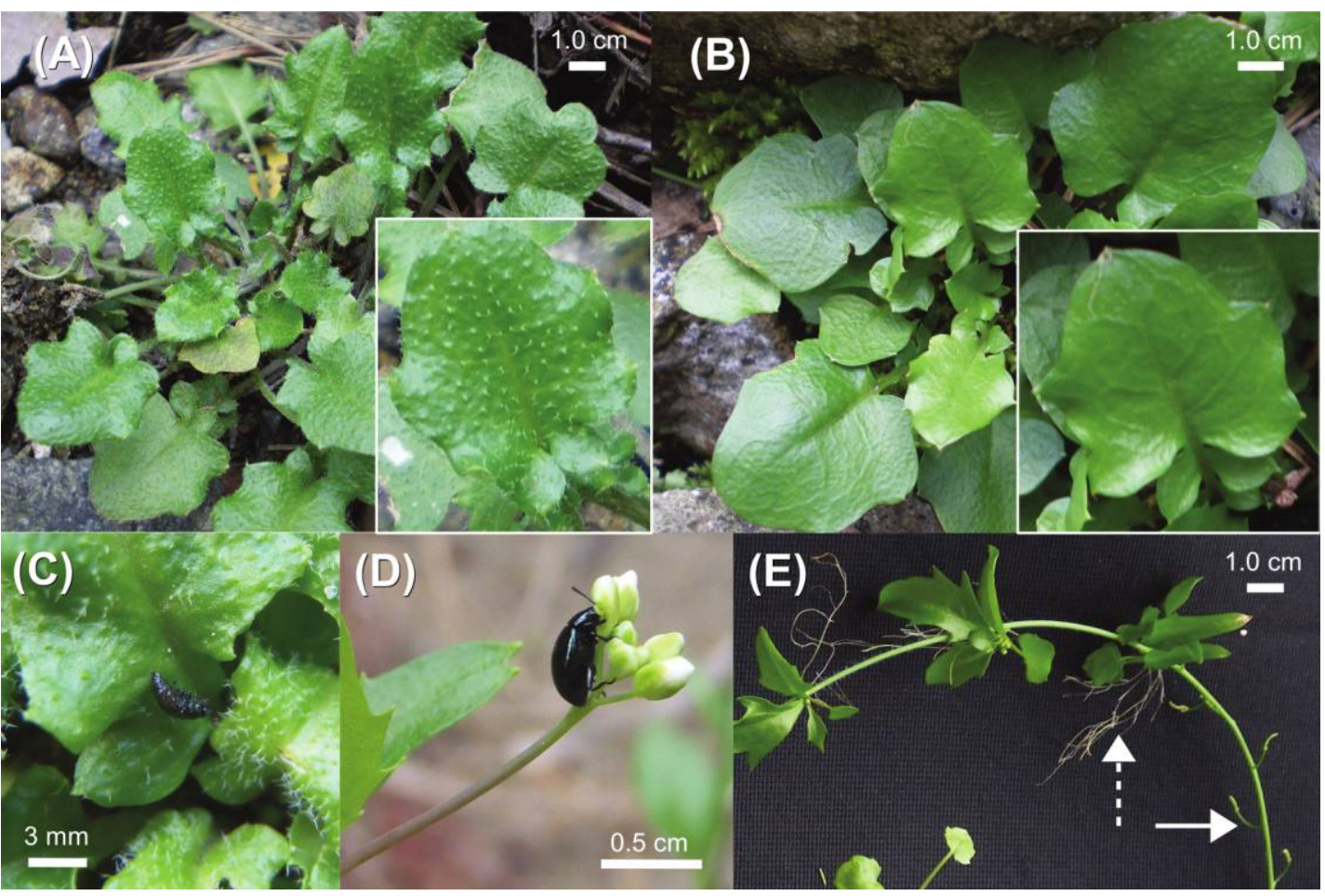

Figure 1: Photographs of Arabidopsis halleri subsp. gemmifera and Phaedon brassicae. A, A hairy plant. $B, \mathrm{~A}$ glabrous plant. $C$, A larva on a hairy plant. $D$, An adult beetle. $E$, A flowering stem of $A$. halleri. Insets in panels $A$ and $B$ focus on a single leaf. Solid and dashed arrows in panel $E$ indicate pedicels and clonal rosettes of $A$. halleri, respectively.

We haphazardly selected 80 circular patches (diameter $1.0 \mathrm{~m},>3 \mathrm{~m}$ apart) in 2013. Trichome phenotype (hairy or glabrous), leaf damage, number of flowers, and length of the largest leaf (hereafter, maximum leaf length) were recorded for all plants in a series of patches. The proportion of leaf area lost to herbivory was visually scored by a single observer as $0(<12.5 \%), 0.25( \pm 0.125), 0.5( \pm 0.125)$, $0.75( \pm 0.125)$, or $1.0(>87.5 \%$ and $<100 \%)$ for each leaf separately. The maximum leaf length was measured as an indicator of plant size, as it reflects the biomass of A. halleri individuals (Sato and Kudoh 2016). The number of flowers was assessed by the number of peduncles remaining on flowering stems. We focused on local interaction in 1-m-diameter patches, because the number of plants within circular patches approached a plateau with increasing patch size (mean \pm $\mathrm{SE}=3.0 \pm 0.3,7.1 \pm 1.0$, and $8.8 \pm 1.3$ plants occurred within patches of $0.5,1$, and $3 \mathrm{~m}$ in diameter, respectively; Sato et al. 2014). The center of each patch was marked using a steel anchor (15 cm in depth) and checked twice a year to survey the same patches among study years. The surveys were conducted once a year during mid to late May (on May 21-23, 2013; May 20-23, 2014; May 19-22, 2015; May 13-15, 2016). We conducted these surveys after the peak abundance of $P$. brassicae at which leaf damages were fully reflected to plants. At the peak abundance, we found $0.2 \pm 0.1$ beetles per plant (mean $\pm \mathrm{SE}, n=100$ plants randomly chosen across a population). We observed 110 beetles during the survey (which was conducted after the peak abundance of beetles), but there was no significant correlation between the number of beetles and the frequency of glabrous plants in a patch $\left(r=0.003, t_{322}=\right.$ $0.05, P=.96)$. We established three to 10 new patches every year to maintain a sample size of 750-850 individual plants per survey. A total of $80-84$ patches were surveyed every year.

For the field survey data, we used generalized linear mixed models (GLMMs) to test whether plant damage and fitness depend on the frequency of neighboring plant morphs. These GLMM analyses were separately performed for the two response variables, the leaf damage score, and the number of flowers. A cumulative error structure was applied to the ordinal response of the leaf damage score from 0 to 1.0, and Poisson error was used to fit the count data of the number of flowers. The fixed effects were the trichome phenotype, the frequency of glabrous plants in a patch ( = number of glabrous plants divided by total number of plants), and the total number of $A$. halleri plants in a patch. To determine the frequency or density dependence in the 
response variables, we incorporated interactions between the trichome phenotype and the frequency of glabrous plants or the total number of plants. The maximum leaf length was used as a covariate in the cumulative error structure for the leaf damage score and as an offset term in the Poisson structure for the number of flowers to consider size-dependent damage and reproduction, respectively, in the statistical model. The study year and patch ID (nested below the study year) were incorporated as random effects. The spatial distance among patches was significantly correlated with neither leaf damage $(r=-0.01, P=.59$ with 9,999 permutations) nor the number of flowers ( $r=0.04$, $P=.26$ ); therefore, spatial autocorrelation was not considered in the GLMMs. We performed likelihood ratio tests to first examine interactions and then compare models with or without single terms. We then estimated coefficients of the fixed effects of the frequency of glabrous plants, total number of A. halleri plants in a patch, and maximum leaf length in GLMMs. These explanatory variables were standardized to 0 mean and variance of 1 to standardize coefficients in a single model. Average trend lines were represented by the middle category (score from 0.25 to 0.5 ) in cumulative models. For figure presentation, the proportion of leaf area lost in a whole plant (hereafter, leaf damage) was calculated by averaging the damage score of leaves. All statistical analyses were performed using $\mathrm{R}$ version 3.2.0 ( $\mathrm{R}$ Core Team 2015). We used the clmm function with the equidist option (in the ordinal package; Christensen 2015) for the cumula- tive error structure and the glmer function (in the lme4 package; Bates et al. 2015) for the other error structures.

We additionally examined whether frequencies of rarer morphs increased in a series of plant patches between successive years. We used a linear mixed model (implemented in the lmer function in R) where the study years were assigned to a random effect. The response variable was the current year's frequency of glabrous plants in a patch, and the explanatory variables were the past year's frequency of glabrous plants and the past total number of $A$. halleri plants. The study site was hit by a typhoon in autumn 2014, resulting in the loss of plants from 16 patches; thus, the sample size was decreased to 64 patches in the data of frequency changes from 2014 to 2015 . Negatively frequencydependent dynamics of the two morphs were determined by slope coefficients less than unity in the linear regression (Sokal and Rohlf 2012).

\section{Mesocosm Experiment}

To test whether the leaf beetles cause a rare-morph advantage in defense and fitness between hairy and glabrous plants (fig. 2), we manipulated the neighborhood conditions of hairy or glabrous plants and the presence or absence of herbivores. We constructed 48 conical plots using gardening poles and wires (diameter $0.75 \mathrm{~m}$ and height $0.7 \mathrm{~m}$ ) to simulate field patches. These plots were separately enclosed by a nonwoven agricultural net ( $90 \%$ light transmittance).

(A) Experimental setting

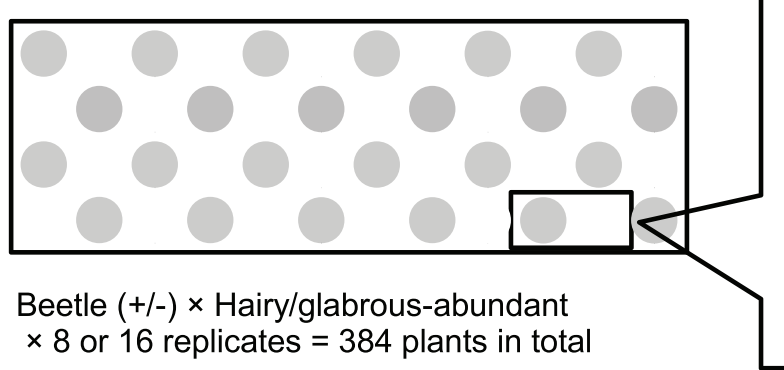

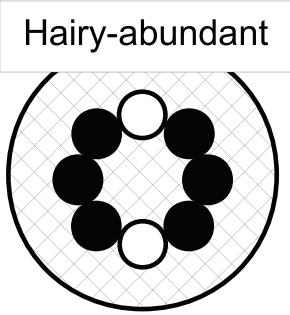

•:Hairy plant $\circ:$ Glabrous plant
Glabrous-abundant

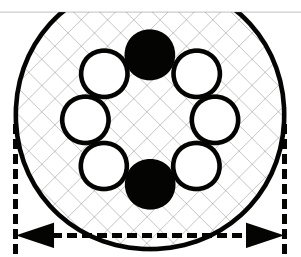

$0.75 \mathrm{~m}$ $\times 8$ or 16 replicates $=384$ plants in total

(B) Time-line procedure

Dec. 2014

Plant stage: Seedling Procedure: Transplant
3.5 month

Bolting

Transfer to the plots
4.5 month

6.0 month (May 2015)

Flowering initiation

Beetle release
Flowering termination End of experiment

Figure 2: Schematic explaining the setting $(A)$ and procedure $(B)$ of the mesocosm experiment. $A$, Two neighborhood conditions were set: hairy: glabrous plants $=6: 2$ and 2:6 individuals (referred to as hairy-abundant and glabrous-abundant conditions, respectively). These two conditions were arranged in a checkered manner (gray = hairy-abundant; white = glabrous abundant), and 16 or eight replicates were assigned to beetle-present (solid circle) or beetle-absent (dashed circle) treatments, respectively. $B$, A horizontal arrow indicates the time series from the start (left, December 2014) to the end (right, May 2015). Plant stage and experimental procedure are described in the first and second row of a square, respectively. The timing of each procedure is shown above each square ( $x$ month after transplanting). 
The experiment was carried out in a greenhouse $(10 \mathrm{~m} \times$ $3.5 \mathrm{~m}$, with a height of $2.0 \mathrm{~m}$ ) located in an open space outside the Center for Ecological Research, Kyoto University $\left(34^{\circ} 58^{\prime} \mathrm{N}, 135^{\circ} 57^{\prime} \mathrm{E}\right.$, altitude $\left.100 \mathrm{~m}\right)$. We removed all weeds on the ground of the experimental area and covered the area with weed-protecting sheets. The greenhouse was covered with an agricultural plastic sheet (thickness $0.1 \mathrm{~mm}$ ). Air temperature was not controlled and ranged from $5^{\circ}$ to $40^{\circ} \mathrm{C}$ during the experiment, which was comparable with that in the field site at plant height level $\left(8^{\circ}-33^{\circ} \mathrm{C}\right)$. Photosynthetically active radiation and the red:far red ratio inside the greenhouse were $1,200-1,400 \mu \mathrm{mol} / \mathrm{m}^{2} / \mathrm{s}$ and $1.0-1.2$ on a sunny day, respectively (LI-190 Quantum Sensor; LI-COR, Lincoln, NE).

We simulated a natural plant population by using seeds collected from multiple lineages of field-grown plants. We initially prepared approximately 600 plants in the greenhouse during the winter season of 2014-2015. Matured seeds were collected from 10 hairy and glabrous plants randomly sampled in the field site. Seeds were germinated on Petri dishes (diameter $9 \mathrm{~cm}$, depth $1.5 \mathrm{~cm}$ ) filled with moistened quartz sand in an environmental chamber under 12L:12D with $25^{\circ}$ and $15^{\circ} \mathrm{C}$ air temperature, respectively, with $>80 \%$ of seeds germinating successfully. During late November 2014, we transplanted one or two seedlings with two to four leaves to a plastic pot (diameter $9.0 \mathrm{~cm}$, depth $8.0 \mathrm{~cm}$ [Y-pot; Sakata Seed, Yokohama, Japan]) filled with seedling-raising compost (Takii, Kyoto, Japan; fertilizer contents, $\mathrm{N}: \mathrm{P}: \mathrm{K}=$ 320:210:300 mg/L, respectively). To prevent herbivory, these potted plants were placed inside transparent plastic cases $(75 \mathrm{~cm} \times 45 \mathrm{~cm} ; 28$ pots $\times 22$ cases in total $)$. The location of the cases was randomized every 2 weeks. Seven weeks after transplanting, we removed smaller plants (evaluated by maximum leaf length) from each pot to prevent competition within the pot.

Two months after transplanting, we transferred potted plants to the experimental plots. Eight individual plants were assigned to each plot, with contrasting numbers of hairy: glabrous plants $=6: 2$ or $2: 6$ individuals (referred to hereafter as the hairy-abundant or glabrous-abundant conditions, respectively; fig. 2). These two conditions simulated the field density with a higher and lower morph frequency $(=10.0$ plants per patch on average; 0.48 frequency of glabrous plants across a population). The eight potted plants were circularly arranged within a plot, and rare morphs were placed with half the rare morphs on each side of the circle (fig. 2). The compass position of rarer plants was randomized to minimize bias in light conditions between rare and abundant morphs. We did not use small plants $(<40 \mathrm{~mm}$ in maximum leaf length) to control for initial plant size. Maternal plant families were randomly assigned among replicates. The final samples consisted of two to 57 plants from 20 maternal families due to variation in plant size and seed number. Sixteen replicates were established for the beetle-present treatments $(8$ plants $\times 2$ frequency conditions $\times 16$ plots $=$ 256 plants), while the remaining 16 plots were assigned to the beetle-absent condition ( 8 plants $\times 2$ frequency conditions $\times 8$ plots $=128$ plants). Plots were placed in $4 \times$ 12 plots in the greenhouse. Water was supplied every 3 or 4 days during the experiment. None of the plants was damaged by herbivores before the experiment.

In the beetle-present condition, we released 12 adult beetles (six males and six females) per plot for the beetlepresent group 4.5 months after transplanting (March 2015). The sex of adult beetles is determined from their body size (adult males have abdomen heights and widths $<0.5 \mathrm{~mm}$ ). The number of beetles per plant was on average $1.4 \pm 0.8$ $( \pm \mathrm{SD})$ for plants inhabited by beetles in the natural population, and therefore we applied the condition of 1.5 beetles per plant to simulate field plants attacked by P. brassicae. The beetles were introduced when plants started flowering because $P$. brassicae is most abundant and influential on plant fitness during the flowering season (Kawagoe and Kudoh 2010). We evaluated herbivory load (leaf damage and herbivore abundance) and plant fitness (flower and clone production) at the end of reproduction (1.5 months after the release of beetles). Leaf damage was scored in the same manner as in the field survey. We counted $P$. brassicae adults and larvae on each individual plant. This herbivore abundance was recorded weekly after larvae had been observed for the first time (2-7 weeks after the release of adult beetles) to evaluate the cumulative herbivory load on plants. We counted flowers as the number of pedicels. Fruit set was not evaluated due to the self-incompatibility of $A$. halleri. The main and lateral meristems of the flowering stem of $A$. halleri produce roots and turn into clonal rosettes (fig. $1 E$ ); thus, clonal meristems were counted to assess clonal reproduction.

To test whether the leaf damage and plant fitness depend on the neighborhood condition, we analyzed the mesocosm experiment data using GLMMs in the same manner as the field survey data. The leaf damage score (with a cumulative error) and the number of beetles, clonal meristems, and flowers (Poisson error) were separately analyzed as the response variable. We used likelihood ratio tests to examine the fixed effects of trichome phenotype and neighborhood condition. Interactions between these two fixed effects were included to test frequency dependence in the response variables. The plot ID and maternal plant ID were included as random effects to consider variation among plots and maternal families. The maternal plant ID was included to deal with unevenness in the number of individual plants among maternal families. The maximum leaf length before flowering was used as a covariate for leaf damage data and as an offset term for the count responses. Pairwise likelihood ratio tests were also performed with sequential Bonferroni correction to determine significant differences among the two phenotypes and the two neighborhood conditions. These analyses were conducted 
separately for the beetle-present or beetle-absent treatments. The field and mesocosm data are deposited in the Dryad Digital Repository: http://dx.doi.org/10.5061/dryad.53k2d (Sato and Kudoh 2017).

\section{Results}

\section{Field Survey: Frequency-Dependent Damage} and Patch Dynamics

Leaf damage to hairy and glabrous plants depended on the frequency of neighboring glabrous plants, as confirmed by a significant interaction between the trichome phenotype and the frequency of glabrous plants $\left(\mathrm{LR}-\chi^{2}{ }_{1}=67.5, P<\right.$ $10^{-16}$; see table $\mathrm{A} 1$ in the appendix; tables $\mathrm{A} 1-\mathrm{A} 3$ and the appendix are available online). Leaf damage to hairy plants decreased as the frequency of glabrous plants increased in a patch (table 1, pt. A; fig. $3 A$ ). In contrast, leaf damage to glabrous plants was not significantly correlated with the frequency of glabrous plants (table 1, pt. A; fig. 3B). The leaf damage also depended on the total number of plants (trichome $\times$ number of plants interaction, LR- $\chi^{2}{ }_{1}=13.6$, $P=.0002$; tables A1, 1, pt. A): leaf damage to hairy plants increased as the total number of plants increased in a patch (Wald test, $Z=2.09, P=.04$; table 1 , pt. A), whereas no significant relationships were detected between damage on glabrous plants and the total number of plants $(Z=1.04$, $P=.30$; table 1 , pt. A).

The number of flowers was also frequency dependent, as shown by a significant interaction between the trichome and frequency of glabrous plants (table 1, pt. B; see table A1 and fig. A1 in the appendix; figs. A1, A2 are available online). Although the flower production was negatively but not significantly correlated with the frequency of glabrous plants for both the hairy and glabrous data sets $(Z=-1.6$ and $-1.7, P=.11$ and .09 , respectively; table 1 , pt. B), the positive coefficient of trichome $\times$ frequency interaction indicated that the number of flowers of hairy plants relative to glabrous plants increased as the frequency of glabrous plants increased (table 1, pt. B; fig. A1). The flower production of the two morphs also depended on the plant density (table 1, pts. A, B). The negative coefficient of trichome $\times$ number of plants interaction indicated that the relative number of flowers of hairy plants decreased as the total number of plants increased (table 1, pt. B). In addition, we separated the field data as beetle-

Table 1: Results of the field survey on leaf damage (A), flower production (B), and temporal variation in the morph frequency (C)

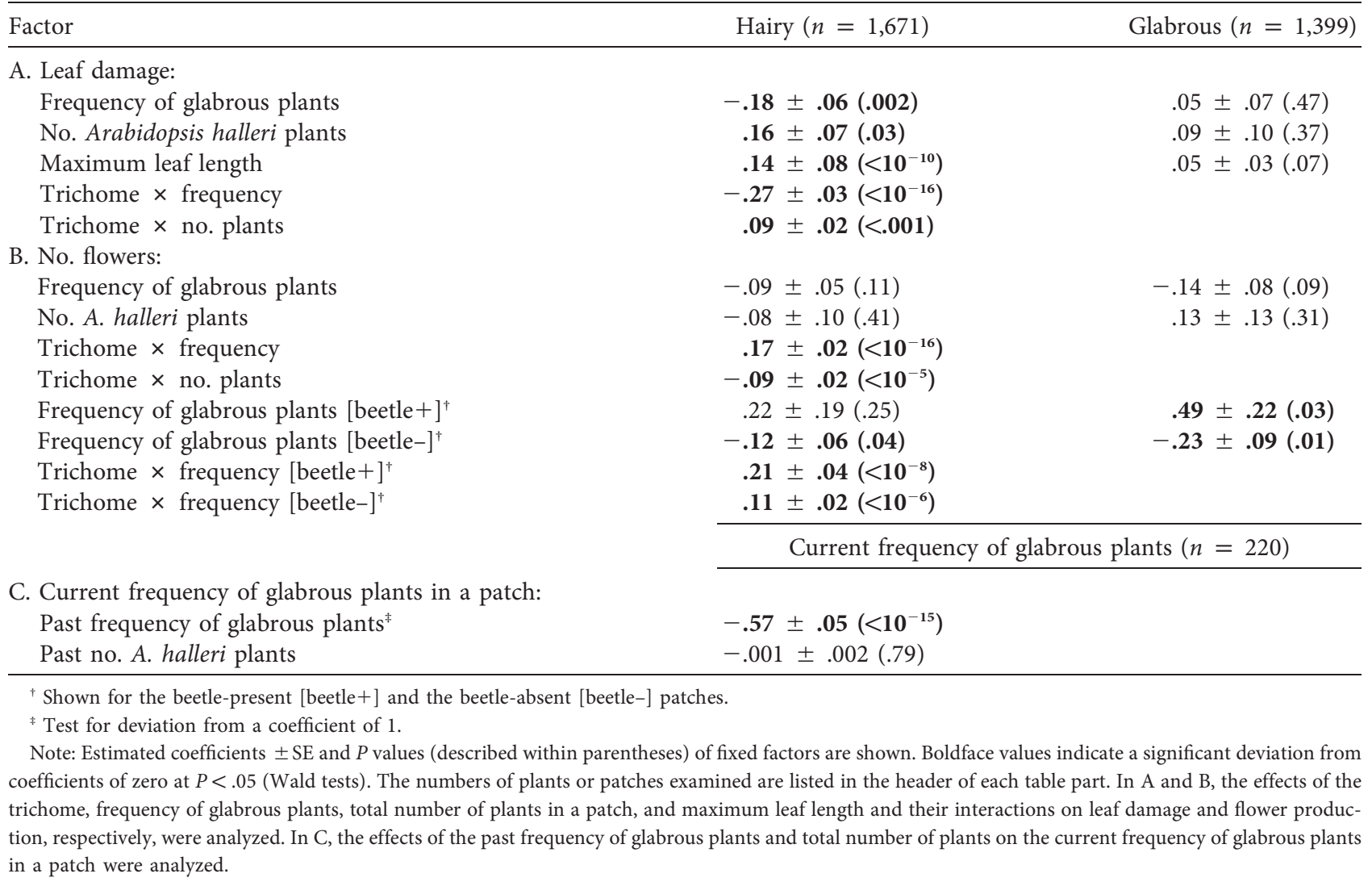


(A) Damage on hairy plants

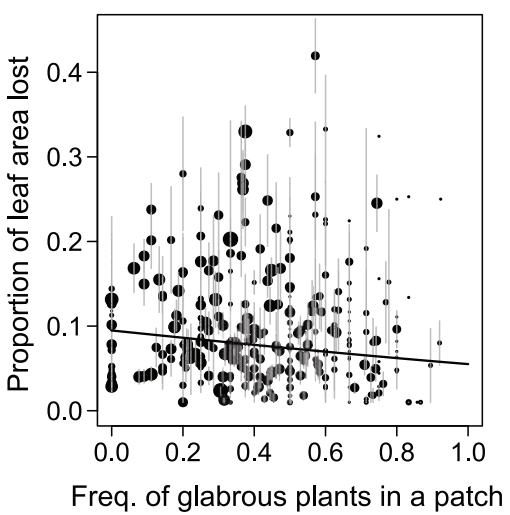

(B) Damage on glabrous plants

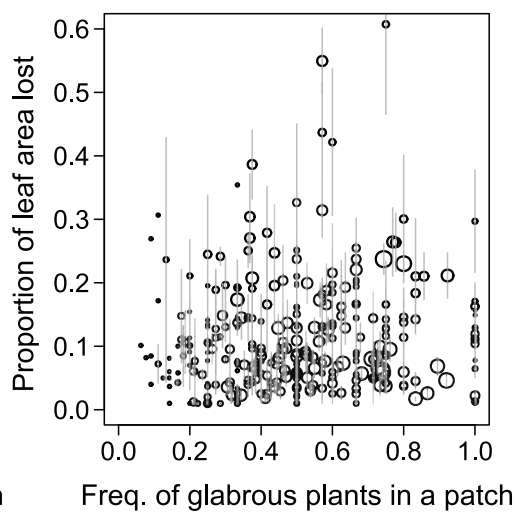

(C) Morph-frequency variation

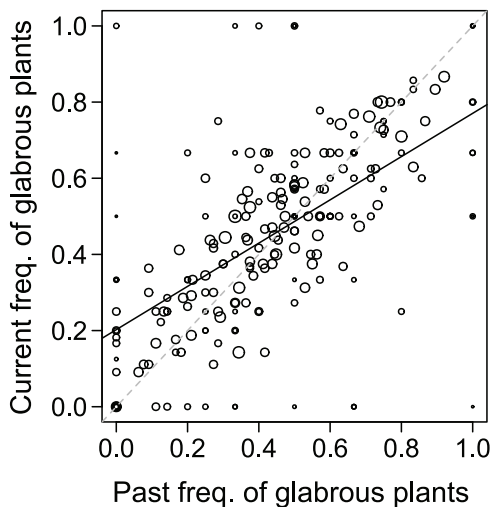

Figure 3: Frequency-dependent leaf damage and temporal variation of hairy and glabrous plants in the natural population. A single point represents a plant patch, and its size corresponds to the number of plants in a patch. Panels $A$ and $B$ focus on the proportion of leaf area that was lost from hairy (filled circles) and glabrous (open circles) plants plotted against the frequency of glabrous plants in a patch. Bars indicate standard errors of the mean of the raw data for damage. A solid line represents a significant trend line by a generalized linear mixed model. Panel $C$ shows the frequencies of glabrous plants in current years plotted against past frequencies in a patch. Solid lines indicate linear predictions, while dashed lines indicate no frequency changes between years $(y=x)$.

present or beetle-absent patches because of a significant three-way interaction among the trichome, frequency, and presence or absence of beetles $\left(\mathrm{LR}-\chi^{2}{ }_{1}=5.03, P=.02\right.$; table A1). There were negative correlations between the flower production and the frequency of glabrous plants in beetle-absent patches, but these correlations were positive in beetle-present patches (table 1, pt. B; fig. A1). The trichome $\times$ frequency interaction had a more positive coefficient on the flower production in the presence than in the absence of beetles (table 1, pt. B; fig. A1). These results provide partial evidence of negative frequency dependence in the relative fitness under the beetle-present condition.

The frequency of glabrous plants in a patch increased when the frequency had been small in the previous year. In contrast, the frequency of glabrous plants decreased when the frequency had been large in the previous year (fig. 3C). This tendency indicates that the frequency of rarer morphs is restored to an intermediate level over time. This result was confirmed by the linear prediction, which had a slope coefficient that was significantly smaller than 1 (coefficient $\pm \mathrm{SE}=-0.57 \pm 0.05$; test for a slope coefficient, $t_{216}=-8.35, P<10^{-15}$; table 1 , pt. C). The past number of Arabidopsis halleri plants in a patch had no significant relationship with the current frequency of glabrous plants (table 1, pt. C).

\section{Mesocosm Experiment: Rare-Morph Advantage in Plant Damage and Reproduction}

Leaf damage and the number of beetles on the two plant morphs depended on the neighborhood condition (indicated by a significant trichome $\times$ neighborhood interac- tion: LR- $\chi^{2}{ }_{1}=134$ and 32.9, $P<10^{-16}$ and $P<10^{-8}$ for the damage and beetles, respectively; table A2). Hairy plants were damaged less than glabrous plants under glabrous-abundant conditions $\left(\mathrm{LR}-\chi^{2}{ }_{1}=129, P<10^{-15}\right.$; table A3). In contrast, leaf damage showed no significant difference between the two morphs under hairy-abundant conditions ( $\mathrm{LR}-\chi_{1}{ }_{1}=0.8, P=.74$; table A3; fig. $4 A$ ). Consistent with the results on leaf damage, fewer beetles were detected on hairy plants compared to glabrous plants under hairy-abundant conditions (LR- $\chi_{1}{ }_{1}=28.1, P<10^{-5}$; tables A2, A3; fig. 4A). This pattern was observed for both larvae and adults (fig. A2), with larvae being more abundant than adults (mean \pm SE $=2.5 \pm 0.15$ larvae, $0.30 \pm$ 0.03 adults). The neighborhood dependence of leaf damage was still significant (i.e., trichome $\times$ neighborhood interaction, LR- $\chi^{2}{ }_{1}=134, P<10^{-16}$; table A2), even though initial maximum leaf length also had a significant effect on leaf damage $\left(\mathrm{LR}-\chi^{2}{ }_{1}=89.2, P<10^{-16}\right)$. No plants died due to herbivory during the experiment.

At the end of the experiment, the rarer plant type had a larger number of clonal meristems and flowers than the major plant type in the presence of beetles (indicated by a significant trichome $\times$ neighborhood interaction: $\operatorname{LR}-\chi^{2}{ }_{1}>119$, $P<10^{-15}$; table A2; fig. $4 A$ ). Under beetle-absent conditions, no rarity advantage was found in the number of flowers and clonal meristems between hairy and glabrous plants (fig. 4B). Glabrous plants produced more flowers and clonal meristems than hairy plants in the absence of leaf beetles $\left(\mathrm{LR}-\chi^{2}{ }_{1}>22.8, P<10^{-4}\right.$ for both hairy- and glabrous-abundant conditions; table A3; fig. $4 B$ ), though the number of meristems was marginally affected by an interaction between the trichome phenotype and neighbor- 

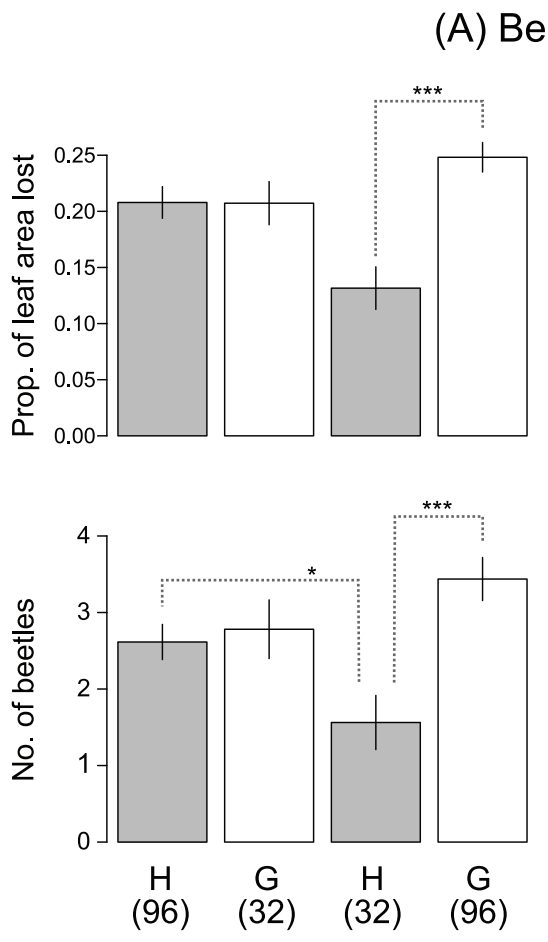

$\mathrm{H}>\mathrm{G}$

$\mathrm{H}<\mathrm{G}$

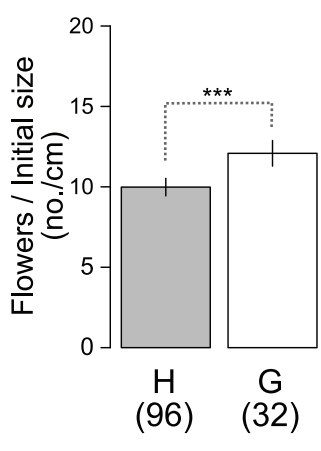

$\mathrm{H}>\mathrm{G}$
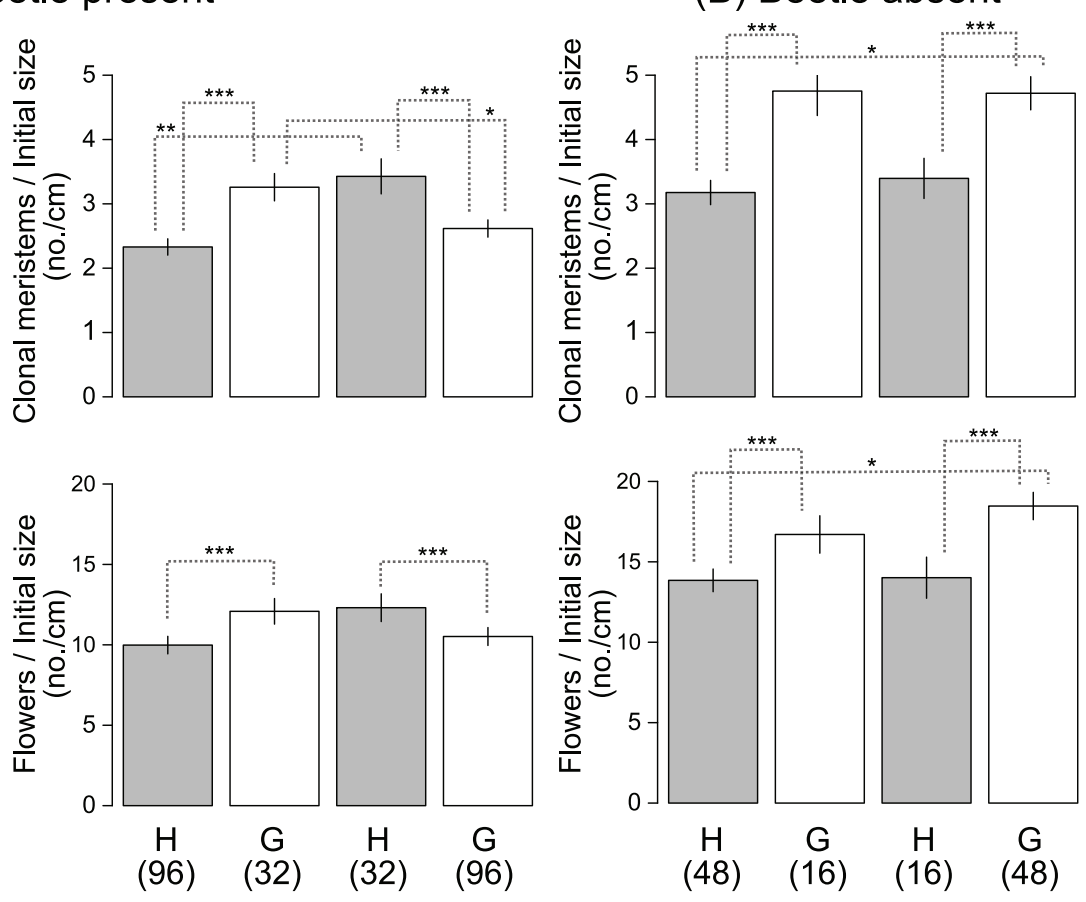

$\mathrm{H}>\mathrm{G}$

$H<G$

Figure 4: Rare-morph advantage with respect to plant damage and fitness in the mesocosm experiment. Leaf damage (proportion of leaf area lost), beetle abundance (number of beetles per plant), and asexual (number of clonal meristems) and sexual (number of flowers) reproduction are shown for hairy $(\mathrm{H}$; gray) and glabrous (G; white) plants under hairy-abundant $(\mathrm{H}>\mathrm{G})$ and glabrous-abundant $(\mathrm{H}<\mathrm{G})$ conditions. The left and right side present the results under beetle-present $(A)$ and beetle-absent $(B)$ treatments, respectively. Bars indicate standard errors of the mean of the raw data. Sample sizes are shown in parentheses. Asterisks represent significant differences among the four groups with likelihood ratio tests (one asterisk $=P<.05$; two asterisks $=P<.01$; three asterisks $=P<.001$ ).

hood condition $\left(\mathrm{LR}-\chi^{2}{ }_{1}=4.16, P=.04\right.$; table A2). This line of results indicates that the presence of Phaedon brassicae causes a rare-morph advantage in herbivory and plant fitness between hairy and glabrous morphs.

\section{Discussion}

Ecological interactions that generate frequency-dependent selection are of considerable interest because of their strong potential to maintain genetic variation (Ayala and Campbell 1974; Greenwood 1984; Barrett 1988; Gigord et al. 2001). Both our field survey and experiment revealed that hairy plants were damaged less when they were the rarer plant type. In the natural population, the flower production was also frequency dependent where hairy plants benefited more from increasing frequency of glabrous plants. The temporal pattern of morph-frequency variation indicates that the frequency of rarer morphs in a series of patches would be restored to an intermediate level over time. More importantly, our mesocosm experiment detected a raremorph fitness advantage between hairy and glabrous plants but only in the presence of Phaedon brassicae. Our results are consistent with the hypothesis that herbivore-mediated interaction promotes the coexistence of hairy and glabrous plants through NFDS. To our knowledge, this research provides the first example illustrating NFDS in an antiherbivore defense trait.

Herbivore-mediated apparent competition has been suggested as a mechanism that stabilizes the coexistence of different plant species (Louda et al. 1990; Pacala and Crawley 1992). Thus far, it has been hypothesized that herbivores facilitate plant coexistence by selectively feeding on abundant but undefended plants when defended plants are less competitive than undefended ones (Pacala and Crawley 1992). Consistent with the results presented here, adults of P. brassicae are known to prefer glabrous leaves of Arabidopsis halleri when hairy leaves are rare (Sato et al. 2014). This escape from herbivory under glabrous-abundant conditions predicts a rare-morph fitness advantage for hairy plants. A defense-growth trade-off may also be supported by our result that glabrous plants produced more flowers and clonal meristems under no herbivory (fig. $4 B$ ) and the 
previous evidence that glabrous plants grew faster than hairy ones did under no herbivory (Sato and Kudoh 2016). As hairy plants no longer had the defense advantage when they became abundant, the defense-growth trade-off predicts that glabrous plants have higher fitness, particularly under hairyabundant conditions. The findings from the P. brassicaeA. halleri system provide a plausible explanation on how a defense-growth trade-off combined with the selective herbivory leads to NFDS between defended and undefended plants.

Although there is evidence for frequency-dependent selection on floral traits in mutualistic interactions (e.g., Gigord et al. 2001; Toräng et al. 2008), our knowledge remains incomplete about NFDS in antagonistic interactions involving a plant defense trait. Some studies on plant-pathogen systems experimentally showed a rarity fitness advantage in cultivated plants (Brunet and Mundt 2000); however, other studies found it difficult to detect NFDS due to simultaneous attacks by pathogens and herbivores in natural populations (Roy 1998; Siemens and Roy 2005). Garrido et al. (2016) have recently tested frequency-dependent selection on complex antiherbivore defenses differing in tolerance and resistance in Datura stramonium but found not negative but positive frequency-dependent selection between the two defensive types. Compared with these previous examples, our study system was relatively simple, because the major herbivorous insect, $P$. brassicae, is a specialist, with $A$. halleri having a known genetic basis of the trichome production. The diamondback moth Plutella xylostella and white butterfly Pieris napi fairly infrequently emerge in the study site (Kawagoe and Kudoh 2010), but their abundance was so low that we could not observe any of these species during our survey. In addition, limited dispersal ability of the flightless leaf beetle enabled us to focus on the local dynamics of plant damage and frequency. Based on previous evidence of NFDS in plants, we suggest that this type of selection is detectable at a spatial scale at which a vector organism is able to freely choose plant morphs without any confounding interactions with other species.

Despite several examples of temporal increases in the frequency of rare morphs in host-parasite interactions (Dybdahl and Lively 1998; Burdon and Thrall 1999; Siemens and Roy 2005), field evidence remains limited for such temporal variation in plant-herbivore interaction. At a local patch scale within a plant population, our study provides partial evidence for such temporal variation in morph frequency of a polymorphic trait. While the flower production of hairy and glabrous plants had no significant slopes along the frequency of glabrous plants, relative reproductive advantage of hairy plants increased as the frequency of neighboring glabrous plants increased (table 1, pt. B; fig. A1). Furthermore, the negatively frequency-dependent benefit for hairy plants was more detectable in the presence than in the absence of beetles. Thus, this weak but negative frequency dependence in the relative fitness partly supports the relevance of herbivore-mediated NFDS in the natural population. The patterns we found were, it is true, weaker in the field data than the mesocosms. One possible explanation for this is that beetle abundance was too low in the field to capture a signature of NFDS via the flower production. Although it is impossible to follow clonal propagation due to its disconnection within a year, our mesocosm results suggest that clonal reproduction also contributes to herbivore-mediated NFDS. Another possible cause of variation in morph frequency is the interpatch migration of hairy and glabrous plants, even though $A$. halleri lacks specialized traits for longdistance seed dispersal. Further investigations on the other fitness components are required to fully understand the fitness consequence of herbivore-mediated interaction and polymorphism dynamics.

We should note that plant density as well as frequency might affect the strength of herbivore-mediated competition (Hambäck et al. 2014; Underwood et al. 2014; Kim and Underwood 2015), since our field survey found density dependence in the leaf damage and flower production (table 1). Damage on hairy plants increased and thereby their flower production decreased as plant density increased in a patch. Thus, the concentration of resources may be important (Root 1973; Underwood et al. 2014), with denser plant patches possibly supporting more herbivores. Because larvae that fed on hairy leaves grew slower than those fed on glabrous leaves (Sato et al. 2014), morph-frequency variation in a plant population might also affect herbivore abundance over a longer timescale. Our survey also found a negative relationship between the flower production and frequency of glabrous plants in the absence of $P$. brassicae but did not detect such a negative relationship in the presence of beetles. Given that glabrous plants save the cost of trichomes under no herbivory (Sato and Kudoh 2016), resource competition might be intense as glabrous plants become abundant in the absence of herbivores. Thus, interactions between herbivory and competition should be examined to obtain a complete understanding about mechanisms maintaining the trichome dimorphism.

In conclusion, our study provides multiple lines of evidence for NFDS acting on antiherbivore defense polymorphism in a natural plant population. Contemporary evolution of antiherbivore defenses has now been reported across several plant taxa (Zangerl et al. 2008; Agrawal et al. 2012; Züst et al. 2012; Sakata et al. 2014); however, these findings raise a further question about the persistence of genetic diversity in plant defense traits under strong natural selection. Existing studies on the maintenance of antiherbivore defense polymorphism discussed nonequilibrium processes such as temporally fluctuating selection (Hare and Elle 2004) or certain genetic constraints (Wise et al. 2009). Based on the evidence for NFDS on a visible and discrete 
trait, this study highlights a stabilizing mechanism for the maintenance of plant defense polymorphisms. Given that dimorphism of defended and undefended plants is widespread in nature (e.g., Hughes 1991; Hare and Elle 2004; Kivimäki et al. 2007; Wise et al. 2009), NFDS on antiherbivore defense traits may be more widespread than previously expected.

\section{Acknowledgments}

We thank T. Kawagoe and J. Sugisaka for providing help during fieldwork. We also thank B. D. Inouye, Y. Sakata, and A. Yamawo for comments and discussions during the study. The manuscript was improved by helpful comments from two anonymous reviewers. This study was supported by the Japan Society for the Promotion of Science (JSPS) through Grant-Aid for Scientific Research (S) to H.K. (26221106) and through Research Fellowships for Young Scientists to Y.S. (15J00400). No conflicts of interests concern this study.

\section{Literature Cited}

Agrawal, A. A., A. P. Hastings, M. T. J. Johnson, J. L. Maron, and J. P. Salminen. 2012. Insect herbivores drive real-time ecological and evolutionary change in plant populations. Science 338:113-116.

Antonovics, J., and N. C. Ellstrand. 1984. Experimental studies of the evolutionary significance of sexual reproduction. I. A test of the frequency-dependent selection hypothesis. Evolution 38:103-115

Augner, M., T. Fagerström, and J. Tuomi. 1991. Competition, defense and games between plants. Behavioral Ecology and Sociobiology 29:231-234.

Ayala, F. J., and C. A. Campbell. 1974. Frequency-dependent selection. Annual Review of Ecology and Systematics 5:115-138.

Barbosa, P., J. Hines, I. Kaplan, H. Martinson, A. Szczepaniec, and Z. Szendrei. 2009. Associational resistance and associational susceptibility: having right or wrong neighbors. Annual Review of Ecologv, Evolution, and Systematics 40:1-20.

Barrett, J. A. 1988. Frequency-dependent selection in plant-fungal interactions. Philosophical Transactions of the Roval Society B 319 473-483.

Bates, D., M. Maechler, B. Bolker, and S. Walker. 2015. Fitting linear mixedeffects models using lme4. Journal of Statistical Software 67:1-48.

Bond, A. B., and A. C. Kamil. 1998. Apostatic selection by blue jays produces balanced polymorphism in virtual prey. Nature 395:594-596.

Brunet, J., and C. C. Mundt. 2000. Disease, frequency-dependent selection, and genetic polymorphism: experiments with stripe rust and wheat. Evolution 54:406-415.

Burdon, J. J., and P. H. Thrall. 1999. Spatial and temporal patterns in coevolving plant and pathogen associations. American Naturalist 153(suppl.):S15-S33.

Christensen, R. H. B. 2015. ordinal: regression models for ordinal data. R package version 2015.6-28. http://www.cran.r-project.org/package $=$ ordinal $/$.
Dybdahl, M. F., and C. M. Lively. 1998. Host-parasite coevolution: evidence for rare advantage and time-lagged selection in a natural population. Evolution 52:1057-1066.

Garrido, E., L. P. Llamas-Guzmán, and J. Fornoni. 2016. The effect of frequency-dependent selection on resistance and tolerance to herbivory. Journal of Evolutionary Biology 29:483-489.

Gigord, L. D. B., M. R. Macnair, and A. Smithson. 2001. Negative frequency-dependent selection maintains a dramatic flower color polymorphism in the rewardless orchid Dactylorhiza sambucina (L.) Soò. Proceedings of the National Academy of Sciences of the USA 98:6253-6255.

Grebe, M. 2012. The patterning of epidermal hairs in Arabidopsisupdated. Current Opinion in Plant Biology 15:31-37.

Greenwood, J. J. 1984. The functional basis of frequency-dependent food selection. Biological Journal of the Linnean Society 23:177-199.

Haldane, J. B. S. 1949. Disease and evolution. La Ricerca Scientifica 19(suppl.):68-76.

Hambäck, P. A., B. D. Inouye, P. Andersson, and N. Underwood. 2014. Effects of plant neighborhoods on plant-herbivore interactions: resource dilution and associational effects. Ecology 95:1370-1383.

Handley, R., B. Ekbom, and J. Ågren. 2005. Variation in trichome density and resistance against a specialist insect herbivore in natural populations of Arabidopsis thaliana. Ecological Entomology 30:284-292.

Hare, J. D., and E. Elle. 2004. Survival and seed production of sticky and velvety Datura wrightii in the field: a five-year study. Ecology 85:615-622.

Hauser, M. T., B. Harr, and C. Schlötterer. 2001. Trichome distribution in Arabidopsis thaliana and its close relative Arabidopsis lyrata: molecular analysis of the candidate gene GLABROUS1. Molcular Biology and Evolution 18:1754-1763.

Hughes, M. A. 1991. The cyanogenic polymorphism in Trifolium repens $\mathrm{L}$. (white clover). Heredity 66:105-115.

Kawagoe, T., and H. Kudoh. 2010. Escape from floral herbivory by early flowering in Arabidopsis halleri subsp. gemmifera. Oecologia 164:713-720.

Kawagoe, T., K. K. Shimizu, T. Kakutani, and H. Kudoh. 2011. Coexistence of trichome variation in a natural plant population: a combined study using ecological and candidate gene approaches. PLOS ONE 6:e22184.

Kim, T. N., and N. Underwood. 2015. Plant neighborhood effects on herbivory: damage is both density and frequency dependent. Ecology 96:1431-1437.

Kivimäki, M., K. Kärkkäinen, M. Gaudeul, G. Løe, and J. Ågren. 2007. Gene, phenotype and function: GLABROUS1 and resistance to herbivory in natural populations of Arabidopsis lyrata. Molcular Ecology 16:453-462.

Louda, S. M., K. H. Keeler, and R. D. Holt. 1990. Herbivore influences on plant performance and competitive interactions. Pages 414444 in J. B. Grace and D. Tilman, eds. Perspectives on plant competition. Academic Press, New York.

Mauricio, R. 1998. Costs of resistance to natural enemies in field populations of the annual plant Arabidopsis thaliana. American Naturalist 151:20-28.

Ôtake, A., and S. Funaki. 1958. The distribution of Phaedon brassicae Baly, with special reference to influences of the dispersal of the adults upon the distribution of their next generation. Bulletin of Shimane Agricultural University 6:107-116.

Pacala, S. W., and M. J. Crawley. 1992. Herbivores and plant diversity. American Naturalist 140:243-260. 
Punzalan, D., F. H. Rodd, and K. A. Hughes. 2005. Perceptual processes and the maintenance of polymorphism through frequencydependent predation. Evolutionary Ecology 19:303-320.

R Core Team. 2015. R: a language and environment for statistical computing. R Foundation for Statistical Computing, Vienna. http:// www.R-project.org/.

Root, R. B. 1973. Organization of a plant-arthropod association in simple and diverse habitats: the fauna of collards (Brassica oleracea). Ecological Monographs 43:95-124.

Roy, B. A. 1998. Differentiating the effects of origin and frequency in reciprocal transplant experiments used to test negative frequencydependent selection hypotheses. Oecologia 115:73-83.

Sakata, Y., M. Yamasaki, Y. Isagi, and T. Ohgushi. 2014. An exotic herbivorous insect drives the evolution of resistance in the exotic perennial herb Solidago altissima. Ecology 95:2569-2578.

Sato, Y., T. Kawagoe, Y. Sawada, M. Y. Hirai, and H. Kudoh. 2014 Frequency-dependent herbivory by a leaf beetle, Phaedon brassicae, on hairy and glabrous plants of Arabidopsis halleri subsp. gemmifera. Evolutionary Ecology 28:545-559.

Sato, Y., and H. Kudoh. 2016. Associational effects against a leaf beetle mediate a minority advantage in defense and growth between hairy and glabrous plants. Evolutionary Ecology 30:137154.

. 2017. Data from: Herbivore-mediated interaction promotes the maintenance of trichome dimorphism through negative frequencydependent selection. American Naturalist, Dryad Digital Repository, http://dx.doi.org/10.5061/dryad.53k2d.

Siemens, D. H., and B. A. Roy. 2005. Tests for parasite-mediated frequency-dependent selection in natural populations of an asexual plant species. Evolutionary Ecology 19:321-338.

Simms, E. L. 1992. Costs of plant resistance to herbivory. Pages 392 425 in R. S. Fritz and E. L. Simms, eds. Plant resistance to herbivores and pathogens: ecology, evolution, and genetics. University of Chicago Press, Chicago.

Sletvold, N., and J. Ågren. 2012. Variation in tolerance to drought among Scandinavian populations of Arabidopsis lyrata. Evolutionary Ecology 26:559-577.
Sletvold, N., P. Huttunen, R. Handley, K. Kärkkäinen, and J. Ågren. 2010. Cost of trichome production and resistance to a specialist insect herbivore in Arabidopsis lyrata. Evolutionary Ecology 24: 1307-1319.

Sokal, R. R., and F. J. Rohlf. 2012. Biometry: the principles and practice of statistics in biological research. 4th ed. Freeman, New York.

Toräng, P., J. Ehrlén, and J. Ågren. 2008. Mutualists and antagonists mediate frequency-dependent selection on floral display. Ecology 89:1564-1572.

Tuomi, J., M. Augner, and O. Leimar. 1999. Fitness interactions among plants: optimal defence and evolutionary game theory. Pages 63-84 in T. O. Vuorisalo and P. K. Mutikainen, eds. Lifehistory evolution in plants. Kluwer, Dordrecht, Netherlands.

Underwood, N., B. D. Inouye, and P. A. Hambäck. 2014. A conceptual framework for associational effects: when do neighbors matter and how would we know? Quarterly Review of Biology 89:1-19.

Wang, X. P., X. M. Zhou, and C. L. Lei. 2007. Development, survival and reproduction of the Brassica leaf beetle, Phaedon brassicae Baly (Coleoptera: Chrysomelidae) under different thermal conditions. Pan-Pacific Entomology 83:143-151.

Wise, M. J., C. G. Yi, and W. G. Abrahamson. 2009. Associational resistance, gall-fly preferences, and a stem dimorphism in Solidago altissima. Acta Oecologica 35:471-476.

Zangerl, A. R., M. C. Stanley, and M. R. Berenbaum. 2008. Selection for chemical trait remixing in an invasive weed after reassociation with a coevolved specialist. Proceedings of the National Academy of Sciences of the USA 105:4547-4552.

Züst, T., C. Heichinger, U. Grossniklaus, R. Harrington, D. J. Kliebenstein, and L. A. Turnbull. 2012. Natural enemies drive geographic variation in plant defenses. Science 338:116-119.

Züst, T., B. Joseph, K. K. Shimizu, D. J. Kliebenstein, and L. A. Turnbull. 2011. Using knockout mutants to reveal the growth costs of defensive traits. Proceedings of the Roval Society B 278:25982603.

Associate Editor: Stephen B. Heard Editor: Yannis Michalakis

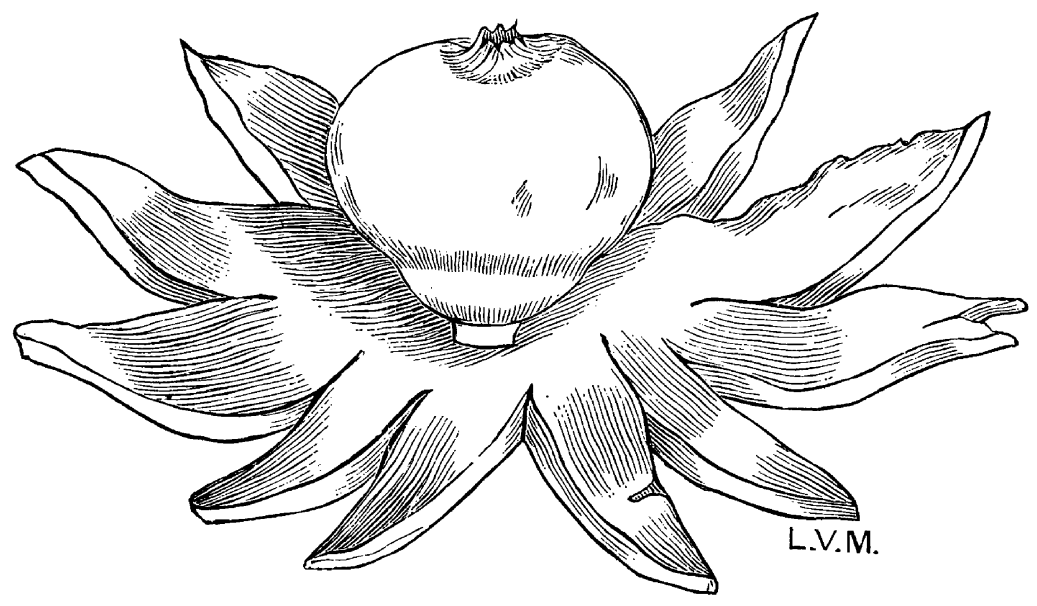

"The Geasters are the most beautiful and attractive of the puff-balls, yet they seem not to be well understood." From "The North American Geasters" by A. P. Morgan (The American Naturalist, 1884, 18:963-970). 